\title{
The I/O transform of a chemical sensor
}

Nalin Katta ${ }^{1}$, Douglas C. Meier ${ }^{2}$, Kurt D. Benkstein ${ }^{2}$, Steve Semancik², and Baranidharan Raman ${ }^{1, *}$

${ }^{1}$ Systems Neuroscience and Neuromorphic Engineering Laboratory, Department of Biomedical Engineering, Washington University in St. Louis, St. Louis, Missouri 63130, United States

${ }^{2}$ Material Measurement Laboratory, National Institute of Standards and Technology, 100 Bureau Drive, Gaithersburg, Maryland 20899

\begin{abstract}
A number of sensing technologies, using a variety of transduction principles, have been proposed for noninvasive chemical sensing. A fundamental problem common to all these sensing technologies is determining what features of the transducer's signal constitute a chemical fingerprint that allows for precise analyte recognition. Of particular importance is the need to extract features that are robust with respect to the sensor's age or stimulus intensity. Here, using pulsed stimulus delivery, we show that a sensor's operation can be modeled as a linear input-output (I/O) transform. The I/O transform is unique for each analyte and can be used to precisely predict a temperature-programmed chemiresistor's response to the analyte given the recent stimulus history (i.e. state of an analyte delivery valve being open or closed). We show that the analyte specific I/O transforms are to a certain degree stimulus intensity invariant and can remain consistent even when the sensor has undergone considerable aging. Significantly, the I/O transforms for a given analyte are highly conserved across sensors of equal manufacture, thereby allowing training data obtained from one sensor to be used for recognition of the same set of chemical species with another sensor. Hence, this proposed approach facilitates decoupling of the signal processing algorithms from the chemical transducer, a key advance necessary for achieving long-term, non-invasive chemical sensing.
\end{abstract}

\section{INTRODUCTION}

Chemical sensing involves detection of a target analyte of interest by measuring a change in a signal that is generated by either the analyte's contact or remote interaction with the sensor. The minimum signal that evokes a measurable output above noise levels is the sensor's detection limit, and the minimum change in the signal levels that can be distinguished is its resolution or sensitivity. In this context, a sensor's operation is akin to a mathematical input/output (I/O) transform[1, 2]. Ideally, the sensing operation will be meaningful if this transform is different for different inputs. Assuming that this hypothesis is true, the proposed formalism faces several practical challenges:

(i) how does one estimate the I/O transform of a sensor?

(ii) how robust is the $\mathrm{I} / \mathrm{O}$ transform to changes of parameters relevant to the sensing task such as identity and intensity of the target signal?

(iii) how invariant is the I/O transform to changes in irrelevant parameters such as sensor age[38]?, and finally

(iv) how reproducible are the I/O transforms for different sensors of equal manufacture?

We examined these issues in the context of chemical sensing with an array of chemiresistive microsensors.

* Corresponding author. Tel: 1-314-935-8538; Fax- 1-314-935-7448; Email: barani@wustl.edu 
Current approaches for chemical sensing have been inspired by the biological principle of using an array of cross-selective chemical sensors to create unique multivariate fingerprints for different odorants. A number of sensing technologies have been proposed for sensitive detection and selective recognition of chemicals[9-20]. Irrespective of the transduction mechanism used, all chemical-sensing approaches must employ a readout mechanism that would allow extraction of meaningful features from the sensor's response. Two strategies seem popular depending on the segment of the response that is assumed to contain most discriminatory information: transient or steady-state responses.

For steady-state response analysis, the ultimate change in the sensor signal after exposure to an analyte is typically the measurement used for further processing [8, 21-24]. In the case of response transients, time-domain features such as sensor's response time constants[25-29] or frequency-domain features such as spectral content[30-32] have been popularly used. In general, it is widely accepted in both artificial and biological chemical sensing systems that the sensor response during transient periods tends to carry richer analyte specific information and therefore can provide better recognition performances[28, 33-44].

Irrespective of the signal readout from the sensor (steady-state vs. transient or time-domain vs. frequency domain), another issue faced by almost all chemical sensors that severely limits their long-term use is the issue of sensor drift, or deviation of the response, over time[3, 7, 26, 45-49]. Drift in artificial chemical sensors has been suggested to primarily be an effect of aging or poisoning of the sensing film[5]. Drift has a profound effect on absolute transducer measurements, making these measures unreliable for long term analyte identification. Therefore, the long-standing need for the development of portable, accurate and precise chemical sensors, which remain viable for extended periods of operation, still remains unmet.

In this work, we propose a simple but elegant approach to characterize the sensor's operation as an I/O transform. The proposed approach exploits the richness of a sensor's response to a temporally structured pulsatile sampling of analytes similar to those used in biological olfaction [37, 38, 41, 50]. It is worth noting that the sensor responses following stimulus onsets, offsets and during their steady-state are all used to characterize a sensor's response to an analyte. We reveal that this approach, to a certain degree, is robust with respect to changes in analyte intensity and to changes due to sensor drift. More importantly, we also show that this approach facilitates generalization between sensors of equal manufacture and therefore provides a way to allow seamless replacement of sensors in a chemical sensing system. The latter problem is fundamental for successful deployment of sensors in commercial applications.

\section{EXPERIMENTAL SECTION}

\subsection{Chemicals used.}

The following five chemicals were used in this study: ethanol (Pharmco-Aaper, Brookfield, CT) ${ }^{*}$, acetone, hexanol, 2-octanol, and 2-octanone (Sigma-Aldrich, St. Louis, MO)*.

\subsection{Analyte delivery and flow system.}

A custom made flow system was used for analyte delivery to the sensor. Liquid chemicals were vaporized in $500 \mathrm{~mL}$ gas washing bottles (Pyrex, Corning, NY) ${ }^{*}$ using zero air (Airgas, St. Louis, MO) ${ }^{*}$. A constant

\footnotetext{
${ }^{*}$ Commercial equipment and materials are identified in order to adequately specify certain procedures. In no case does such identification imply recommendation or endorsement by the National Institute of Standards and Technology, nor does it imply that the materials or equipment identified are necessarily the best available for the purpose.
} 
stream of 0.1 standard $1 /$ min (slm) of zero-grade air was flowed through a bubbler containing a pure liquid analyte. The outflow, assumed to be a saturated vapor, was diluted with a carrier stream of cleaned, dehumidified air flowing at $1 \mathrm{slm}$. A sub-sample of this well-mixed stream $(0.1 \mathrm{slm})$ was further diluted by mixing with a second carrier stream of $0.65 \mathrm{slm}$ of cleaned, dehumidified air. Regardless of whether an analyte was present, a constant airflow of $0.75 \mathrm{slm}$ across the sensor was maintained at all times. The analyte for each trial was selected pseudo-randomly to reduce effects of long-term chemical hysteresis. The analytes were presented in a pulsed fashion as shown in Fig. 1.

For altering analyte concentrations, we varied the relative volume of the saturated output from the bubblers and the first carrier stream, before sub-sampling (i.e during the first dilution stage). Note that only the volume of the first carrier stream was changed; the flowrate through the bubbler was kept constant. The total flow rate of the first carrier stream was varied from a $1 \mathrm{slm}$ flow rate to $0.5,0.75$, or 1.25 slm. The resulting analyte concentrations achieved as a result of this flow modulation are shown in Table S-1.

\section{3 Metal oxide sensor.}

A microsensor array with four individually controllable elements covered by $\mathrm{SnO}_{2}$ sensing films was used in this study. Other sensing elements in the array were left idle. The manufacture of these devices has been thoroughly described previously [17, 51-55]. Briefly, each sensor element is a multilayer, suspended device. From the top, the functional layers are: a polycrystalline $\mathrm{SnO}_{2}$ sensing film, two interdigitated platinum electrodes, an insulating layer, and a polysilicon heater. The operating temperature of the sensor was modulated between $55^{\circ} \mathrm{C}$ and $435^{\circ} \mathrm{C}$. Sensor responses of four copies of the $\mathrm{SnO}_{2}$ microsensors were measured. Each sensor was cycled through 28 temperature steps (Fig. S-1), with each temperature treated as a perturbed-isotherm[56].

Previous work by us [25], has shown that at least two different correlated bands of information were generated at low and high temperatures for most analytes. Therefore, we used a temperature program that sampled a range of temperatures between $55 \mathrm{C}-435 \mathrm{C}$. Consistent with previous results, a cross-correlation of information content obtained at different temperature revealed that information content does change with temperature and in analyte specific manner (Fig. S-2).

Conductance measurements were made at each of the operation steps for each sensor. Each measurement cycle lasted $38 \mathrm{~s}$ as all four sensors used were cycled through 28 temperatures. A logarithm (base 10) was calculated to compress the sensor responses (note that this step is not critical for results reported here). Analysis in this paper was performed using three sensors that lasted the entire data collection period of approximately 7 months.

\subsection{Calculation of the I/O transforms.}

The sensor response $(y)$ at a particular temperature was treated as the dependent variable to be predicted given the most recent stimulus history ( $\vec{x}$; series of 1 and 0 indicating analyte valve open or closed during each measurement; in other words random binary valve states prior to the measurement y). Note, the length of the moving window (i.e. dimensionality of vector $(\vec{x})$ ) is a free parameter. For each sensor, temperature combination:

$$
y=\vec{k} \cdot \vec{x}
$$

where $\vec{k}$ is the transform that maps the stimulus history (the input) onto the sensor's response (the output) for a given analyte. I/O transform for each analyte was calculated using a least squares regression estimation method, where the mean squared error of prediction is defined as:

$$
(\vec{k} \cdot \vec{x}-y)^{2}
$$

A moving window was continuously shifted and the sensor response at the end of the stimulus history was recorded to construct a sensor input matrix $(X)$, and a sensor response vector ( $Y$; a column vector of sensor responses at the end of each stimulus history). A bias term was added to account for average signal 
baseline (DC offset) throughout the experiment. This bias-term was not used in any further analysis as the goal was to focus primarily on the changes in response dynamics. Note that the reconstruction error is minimized when:

$$
\vec{k}=\left(\vec{X}^{T} \vec{X}\right)^{-1} X^{T} Y
$$

i.e. the pseudo-inverse solution. A schematic of this approach is presented in Fig. 2.

For results shown in this manuscript, a stimulus history of eight recent stimulus states was chosen as the window size. This selection was based on the minimum window length for which the error of reconstruction converged (Fig. S-3). This was done to provide a sufficient tradeoff between minimizing reconstruction error and reducing over-fitting to the training data.

Note that the response of the sensor at each temperature was treated separately. Therefore, we created $28 \mathrm{I} / \mathrm{O}$ transforms for each analyte one for each operating temperature. These are shown in Fig. 2D.

\subsection{Training and Testing Datasets.}

First dataset: The sensor response measurements were collected over a period of a month. Each analyte was presented in a random binary pulsatile sequence (analyte ON and OFF) as shown in Fig. 1. A single pulsatile sequence run resulted in 140 sensor response measurements ( $\sim 40$ with analyte ON period and $\sim 100$ analyte OFF period). The training data set consisted of 17 such measurement sequences; 3 for acetone, 3 for ethanol, 4 for hexanol, 4 for 2-octanol, and 3 for 2-octanone. Note that this was the training data used for generating the $\mathrm{I} / \mathrm{O}$ transforms shown in Fig 2, and 3.

Second dataset: The sensor array was purposely aged for a period of two months during which it was intermittently operated with exposure to the same analytes. Subsequent to the aging phase, a second experimental run, lasting a 2-3 weeks of data collection, was used to validate our approach. The second experimental run consisted of similar sequences as in the training period. 20 such measurement sequences were made (4 for each analyte). This data was used as the testing data for quantifying performance of the models generated using the first training dataset (refer Fig. 4).

Third dataset (Concentration dataset): To further assess the limits of our methodology a third dataset using a subset of the analytes (acetone, ethanol, hexanol, and 2-octanol) was presented at varying concentrations. Note that this third dataset was collected 3 months after the collection of the second dataset. This dataset consisted of 13 measurement sequences; 4 for Acetone, 3 for Ethanol, 3 for Hexanol, and 3 for 2Octanol. Also note that the dataset collected during the previous data collection phase (i.e. second dataset) were used to create the I/O transforms for classifying these responses (Fig. 6).

\section{6 Dimensionality reduction and classification.}

A linear principle component analysis (PCA) was used for visualizing the I/O transforms associated with each analyte. The I/O transforms or response filters were projected onto eigenvectors corresponding to the three largest eigenvalues of the correlation matrix. Only the training data and their corresponding transforms were used for determining the PCA axes. Both training and testing datasets were projected onto the same axes to aid visualization and comparison.

In order to classify testing data, we implemented a hierarchical, recursive approach. In this scheme, we treated one m-class classification problem into a series of m binary classification problem. The algorithm can be summarized as follows:

1. Compute the $\mathrm{I} / \mathrm{O}$ transform of the test analyte that needs to be classified

2. Perform PCA to reduce dimensionality of the filter (Note that this step was performed for visualization, but not strictly necessary for the approach).

3. Compute the mean centroid of the dimensionally reduced $\mathrm{I} / \mathrm{O}$ transform of each training analyte 
4. Repeat until done

a. Based on pairwise distances between the mean $\mathrm{I} / \mathrm{O}$ transforms, find the analyte that is farthest from all others (i.e. the analyte with the most unique I/O filter shape; in Fig. 5 this is ethanol).

b. Project all training and test data onto a difference of mean plane between the centroid of the farthest analyte and the centroid of the closest analyte (for ethanol the closest response cluster is acetone).

c. Use a nearest neighbor classifier to assign the test sample to one of the two classes: farthest analyte vs. rest of training

d. If assigned to farthest analyte, then stop recursion.

e. If only two training analytes remain, then classify the test sample using the label of the nearest neighbor in the training sample.

f. Else, remove the samples from the farthest analyte from further consideration and repeat steps a-e.

\section{RESULTS}

\section{$\underline{3.1 \text { Responses of a chemical sensor to pulsatile stimuli }}$}

We began by examining the response of a metal-oxide chemiresistor to a few analytes that varied in their functional group, carbon chain length and vapor pressure. Unlike most chemical sensing studies, we delivered stimuli in a pulsatile fashion to exaggerate the response transients. The pulses delivered varied in duration, (1 to 3) min, and inter-pulse interval, (1 to 4) min. The mean response of the sensor ( \pm standard deviation (S.D.)) at one particular operating temperature $\left(435^{\circ} \mathrm{C}\right.$; see Fig. S-1) is shown in Fig. 1. Note that sensor responses to the exposed gas were measured at twenty eight operating temperatures (Fig. S-1).

In general, irrespective of the operating temperatures, we found that all gases used increased sensor's conductance (i.e. all were reducing gases) with the absolute magnitude of the response being greater for ethanol and acetone (blue and green) compared to the others. We found that the pulsed stimulus sequence emphasized differences in the transient responses generated by different analytes. During a relatively lengthy pulse, there was a discernable difference in time to peak response between different analytes (Fig. 1, inset 1). Similarly, when the inter-pulse interval was reduced, responses to the non-leading pulses decreased substantially for all analytes (Fig. 1, inset 2). Note that the magnitude of response reduction was analyte specific. Hence, we hypothesized that the pulsed mode of stimulus exposures could enhance discriminability between analytes. Furthermore, since some of the response dynamics were governed by the differences in stimulus dynamics, we expected these transient portions to remain invariant to sensor drift, consistent with previously shown results[57].

\subsection{Chemical sensing as an I/O transform}

For any given analyte, and at any particular point in time, the state of the valve delivering the stimulus (open or closed) and the sensor's response were both known. We viewed the sensor's operation as a transform that when applied to the stimulus state produced a corresponding response. If the sensor was extremely rapid and there was no hysteresis, then the I/O transform could be regarded to be instantaneous. However, this was not true for our sensor and for most chemical sensors studied in general. Therefore, we assumed that the sensor response at any given time is not instantaneous but a weighted linear function of the recent stimulus history. Note that this I/O operation or transformation must still be unique for each analyte in order for the sensor to generate unique fingerprints.

To estimate the linear transform from the training data, we recorded the stimulus history, as determined by the valve state, in a given time window $(\vec{x})$ and the sensor response at the end of this period ( $y$; a scalar value). For example, the following valve-states for last eight measurements [closed $_{\mathrm{t}-7}$, open $\mathrm{t}_{\mathrm{t}-6}$, 
open $_{t-5}$, open ${ }_{t-4}$, closed $\mathrm{c}_{\mathrm{t}-3}$, closed $\mathrm{c}_{\mathrm{t}-2}$, closed $\mathrm{d}_{\mathrm{t}-1}$, closed $\left.\mathrm{c}_{\mathrm{t}}\right]$, would result in the following stimulus history vector $[0,1,1,1,0,0,0,0]$. For each stimulus and each trial, or run, we systematically moved the stimulus history window in time to generate a matrix of stimulus histories (X; 8 columns but multiple rows) and a vector of sensor responses measured at the end of each stimulus history (Y; column vector with the same number of components as the rows of $\mathrm{X}$ ). The sensor response model to a given analyte could now be viewed as the optimal linear transform that reconstructs $\mathrm{Y}$ given $\mathrm{X}$ (schematically shown in Fig. 2a). Since this results in an over-determined system of equations (more rows than columns), we calculated the I/O transform to minimize the mean-squared error of reconstruction (i.e. pseudo-inverse solution).

The optimal linear transform $(\vec{k}$; vector of the same dimensions as $\vec{x})$, and the comparison between the actual vs. reconstructed sensor responses for the different analytes used are shown in Fig. 2b and 2c. Note that each component of the I/O transform is a weight for the stimulus/valve state at a particular point in time/history. The overall sensor's response that is reconstructed in this fashion is merely a weighted sum of valve states in the recent past. As can be observed, the actual sensor response (black traces) and the predicted sensor response (red traces) are well-matched for all analytes (see Fig. S-4).

The I/O transform of each analyte for each of the 28 operating temperatures used in the study is shown in Fig. 2D.

\section{3 Recognition of chemicals based on Sensor's I/O transform}

As can be noted from Fig. 2, the I/O transform was unique for each analyte examined. Furthermore, as shown in Fig. 3a, the I/O transform was reliable across multiple training runs for each analyte used. These results suggest that the estimated I/O transform of a sensor may be used as a fingerprint to identify each analyte. To confirm this hypothesis, we first visualized the eight-dimensional I/O transform using principal component analysis (Fig. 3b). Note that each complete training run or trial, resulted in a single estimate of the I/O transform, and therefore is represented as a single point/symbol after PCA dimensionality reduction. Different runs corresponding to a particular analyte generally clustered together and were discriminable from clusters representing other analytes. Therefore, this result suggests that the sensor's I/O transform can indeed be used for analyte recognition.

\subsection{Sensor's I/O transforms are drift tolerant}

As mentioned before, drift can be a major issue with chemical sensors, as it can significantly reduce the viability of sensors operating over an extended period of time. To test the stability of the sensor's I/O transform to each analyte, we aged the sensor for a period of two months (see methods; dataset 2). Subsequent exposures of the same five analytes generated raw sensor responses that were substantially drifted. We note that sensor baseline response decreased and the sensor response magnitude varied as a result of this drift (Fig. S-5).

We made a qualitative comparison of sensor response profiles before (training dataset) and after (testing dataset) aging using a linear principle component analysis (Fig. S-6a). Note that sensor response from the training dataset is indicated using circles, whereas raw sensor responses during the validation phase are identified as squares. As can be noted, although groupings within datasets are well-defined, drift in sensor responses across datasets shifted the overall response profiles. Complementing this qualitative visualization analysis, we performed a quantitative nearest-neighbor classification. The overall results from this classification analysis are summarized in the confusion matrix shown in Fig. S-6b. Note that the main diagonal elements were low indicating misclassification. These results qualitatively and quantitatively confirm that the raw responses of the sensor before and after aging were inconsistent.

Next, we compared the I/O transform for different analytes before and after sensor aging (Fig. 4). Despite the evident drift, the I/O transforms were relatively consistent (Fig. 4a). Further, to quantify the performance results we performed a classification analysis (Fig. 5a). We reduced the overall classification problem into a series of two-class discriminations to determine similarity with ethanol vs. others (step1), acetone vs. remaining others (step2), 2-octanol vs. remaining others (step3), and finally hexanol vs. 2octanone (step4). Note that this scheme allows progressive refinement at each step, focusing primarily on 
determining membership to the most distant response cluster (see methods). The discriminations are shown on the right in Fig. 5a. The colored bands indicate regions, of the projection, where analytes would be successfully classified at that step and the gray bands indicate regions of the projection where analytes would be parsed through subsequent stages. The confusion matrix shows that most of the analytes were recognized well above the chance level (17\% to $24 \%$ ), and significantly higher than a direct PCA approach (Fig. S-7).

\subsection{Concentration invariant recognition}

Changes in concentration are also known to alter the sensor response magnitudes [58, 59]. Such response variations can lead to significant overlap in the responses generated by different analytes. Since the I/O transforms of the sensor to each analyte are predominantly focused on the response dynamics, we next examined how robust these were with respect to changes in stimulus intensity. We repeated the experiments with acetone, ethanol, hexanol, and 2-octanol but presented at different intensities.

We found that the I/O transforms were consistent even when the analyte concentrations were changed (Fig. 6a). In addition, we used a correlation based distant metric in our dimensionality reduction (Fig. 6b) to focus primarily in the shape of the I/O transform and not on its magnitude to further reduce sensitivity to variations that might arise due to stimulus intensity. As can be noted, the classification performance was well above the chance levels for all analytes tested in this fashion (Fig. 6c).

To further clarify these results, we scaled the response of a particular analyte using three different values (x1, x2 and x5). Such scaling provided responses that had similar transients and time constants, but with varying magnitude (Fig. S-10). As expected, we found that the I/O transforms obtained for these three responses were identical in shape but differed substantially in their magnitude. Further, we note that a distance metric based on the correlation values would classify these I/O transforms to be identical thereby providing the analytical basis for invariance with respect to the changes in response magnitude.

\subsection{Sensor-invariant analyte recognition}

Finally, we examined how robust the proposed signal extraction approach was across different equivalent sensors that were fabricated together. To examine this, we repeated the same analyses and compared the $\mathrm{I} / \mathrm{O}$ transforms obtained between two sensors. We found that for each analyte, the I/O transforms were surprisingly consistent and training data from one sensor can allow identification of those analytes even when data from a different sensor was used for validation (Fig. 7). Note that these results are largely consistent with using training and testing datasets from the same sensor (refer Fig. 4). This further suggests that the response dynamics are dominated by the stimulus dynamics of the analyte themselves and may provide a robust approach for analyte recognition. Therefore, we expect this approach may also insulate the signal processing approaches to changes happening in the sensor array when damaged sensors are replaced with copies of the same type of sensor.

\section{DISCUSSION AND CONCLUSIONS}

In this work, we have presented a method to characterize a chemical sensor's operation as a linear I/O transform. The input to the sensor is the recent stimulus history, which we defined here as a short timeseries of the valve states ('ON' or 'OFF'). The sensor's response at the end of the stimulus history became the output to be predicted. In this formulation, we showed that for each analyte the sensor's operation became a unique input-output filter or a transform. Such an approach for developing a mathematical model of a dynamical system based on the inputs provided and the outputs measured is commonly recognized as a 'Systems Identification' approach [1, 2, 46, 60-62]. However, in the context of chemical sensing, what is not clear is how to use this system identification approach for recognition/differentiation of different analytes, how stable are these estimated I/O relationships, and how they vary over time, sensors etc. We have carefully explored these issues in this study.

In order for such a scheme to be feasible, it is first important to test whether the impulse response function of a chemical sensor can be reasonably estimated with a random inputs of certain finite length. If 
this assumption is reasonable, then the I/O transform (or alternately impulse response function) computed over one segment of random pulsatile binary inputs should allow prediction of the sensor's response to other random patterns of pulsatile binary inputs as well. We found that this is indeed feasible and the proposed estimation approach is robust and works well even when predicting the response of the sensor to other patterns of random binary input pulses (Fig. 9).

We note that the analyte discriminability was enhanced due to the employment of pulsatile stimulus delivery that enhanced information in the transient sensor responses. The response dynamics were largely driven by the stimuli themselves possibly due to differences in vapor pressure, the physisorption onto the sensor surfaces, etc. Therefore, we found that these analyte-specific transforms were robust, to a certain extent, to variations in analyte intensity and sensor age. The latter feature allowed reliable recognition of the analytes even when the sensor's baseline and the magnitude of stimulus-evoked responses changed over extended periods of operation (see Fig. 1, Fig. S-5, Fig. S-6). Hence, we believe that this approach may provide a drift-invariant analyte recognition scheme, a key requirement towards realizing non-invasive chemical sensing.

The only additional requirement imposed by the proposed technique is the need for active sampling approach as opposed to the typical passive methodology used for gas sensing. While other efforts that have examined the use of a pulsed stimulus delivery protocol for generating information rich datasets from the sensor, these works have either focused on the magnitude of the response [23] from a few short pulses or select features from the signal [63]. However, our approach markedly differs in that we examined not only the signal from stimulus exposure (onset transients and steady-states) but also took into account the transients generated following absence of a gas stimulus (i.e. offset transients). It will be worth noting that, although OFF-responses are informative, they are not considered by most approaches for discriminating analytes.

Such active sampling techniques are routinely used by biological systems to sample the chemical stimuli encountered in their environment ("antennal flicks" in invertebrates[64] or "sniffs" in vertebrates [65]). Active sampling is thought to extend greater control of the stimulus to the system, allowing it to manipulate and define the stimulus dynamics. Previous works have shown that stimulus dynamics can be dependent on the analyte or 'odorant' $[37,40]$ and may be exploited by subsequent processing centers in the brain [38, 41]. Several approaches to generate spatiotemporal sensor response profiles for analytes to enhance their discrimination have also been explored in artificial olfaction [39,63,66]. Our work complements these studies and focuses on development of schemes that take advantage of such rich data streams for the purpose of robust chemical identification.

Using such as scheme in a real-world scenario could be easily achieved by placing a small pump downstream of the sensor and drawing air carrying the encountered analyte over the sensor in a known binary pattern. In this scenario, the I/O transform can be estimated if the random binary stimuli used to control the pump/valve over a period of time and the output of the sensor during this active sampling period are both known. The estimated I/O transform of the currently encountered analyte can then be compared and pattern matched with those of the training analytes for the purpose of recognition.

We note that the proposed scheme has not been optimized for rapid recognition of analytes. We envision changes that could be made to increase the sampling rate and therefore decrease the duration and spacing of pulses used to address this issue in future studies. Furthermore, in agreement with previous studies [26, 67], we found information content across temperatures was redundant. Hence, our temperature programs could be optimized by down-selecting to several high and low temperatures [62, 68-70]. We also found that hysteresis has a pervasive effect in metal oxide gas sensors. In general, our results show that $\mathrm{I} / \mathrm{O}$ transforms of responses collected at temperatures above $250{ }^{\circ} \mathrm{C}$, especially those that occur in the later segments of the temperature cycle, were more consistent across analytes and therefore resulted in higher classification accuracies (Fig. 8 and Fig. S-8). In this study, we did not systematically attempt to take advantage of the hysteresis related effects we observed, which would provide another free parameter for the purpose of optimization. 
Finally, we found that the sensors of equal manufacture generated strikingly similar transforms for a given analyte. This allowed the data obtained from one sensor to be used for recognizing the training analytes with a different sensor. The transferability of the training data is based upon the identification of sensor-independent features for pattern recognition. These can then be used to improve the flexibility of sensor arrays for a variety of application areas, primarily enabling greater longevity once deployed. Our demonstrated approach of pulsatile sampling and I/O transforms has the potential to be a simple, technology-independent technique for achieving this capability. 


\section{VITAE}

Nalin Katta received his B.S. degree in biomedical engineering from the Washington University in St. Louis, in 2010. He is a Ph.D. candidate in the Department of Biomedical Engineering at Washington University, St. Louis, MO, under the guidance of Dr. Baranidharan Raman. His current research interests include sensory and systems neuroscience, bio-mimetic sensors, and device design.

Douglas C. Meier received the B.A. degree in chemistry from Northwestern University, Evanston, IL, and the Ph.D. degree in chemistry from Texas A\&M University, College Station, where he studied the chemical physics of model catalyst systems under the guidance of Prof. D. W. Goodman. He is currently a National Institute of Standards and Technology (NIST) Research Chemist with the Process Sensing Group, NIST, Gaithersburg, MD, applying surface chemistry and thin-film science in the development of advanced chemical microsensor arrays. Dr. Meier was awarded a National Research Council PostDoctoral Research Associateship from the Process Sensing Group, NIST.

Baranidharan Raman received the B.Sc. Eng. degree (with distinction) in computer science from the University of Madras, Chennai, India, in 2000, and the M.S. and Ph.D. degrees in computer science from Texas A\&M University, College Station, TX, in 2003 and 2005, respectively. He is an Assistant Professor with the Department of Biomedical Engineering, Washington University, St. Louis, MO. From 2006 to 2010, he was a joint Post-Doctoral Fellow with the National Institutes of Health and the National Institute of Standards and Technology, Gaithersburg, MD. His current research interests include sensory and systems neuroscience, sensor-based machine olfaction, machine learning, biomedical intelligent systems, and dynamical systems. Dr. Raman is a recipient of the Wolfgang Gopel Award in 2011 from the International Society for Olfaction and Chemical Sensing and a NSF CAREER awardee.

Kurt D. Benkstein received his B.S. degree in Chemistry in 1995 from Iowa State University and his M.S. and Ph.D. degrees in Chemistry from Northwestern University in 1996 and 2000, respectively. He went to the National Renewable Energy Laboratory in 2000 as a postdoctoral researcher to study the relation between film morphology and electron transport in dye-sensitized nanoparticle solar cells. In 2003, Dr. Benkstein joined the National Institute of Standards and Technology as a Research Chemist to study nanostructured materials for chemical sensors.

Steve Semancik is the Project Leader of the Chemical and Bioanalytical Microsensor Program at the National Institute of Standards and Technology (NIST) in Gaithersburg, Maryland. He received his B.S. degree in physics from Rensselaer Polytechnic Institute and his Sc.M. and Ph.D. degrees, also in physics, from Brown University. Dr. Semancik's professional research career began as a National Research Council Postdoctoral Fellow, and has been centered in the fields of surface science and sensor science. His recent work has focused on developing improved nanomaterials for chemical and biochemical sensing, and combining such high performance materials with micromachined platforms to realize advanced microsensor devices and operating modes. He has authored or coauthored more than 150 papers, including multiple reviews, several book chapters, and six patents. Dr. Semancik is an elected Fellow of both the American Physical Society and the American Vacuum Society, has served as a Member of the Editorial Board of two sensor journals, and is a Member of the Steering Committee of the International Meeting on Chemical Sensors. 


\section{AUTHOR INFORMATION}

\section{Corresponding Author}

Barani Raman: barani@wustl.edu

\section{Author Contributions}

Experiments and analysis were designed by NK and BR and were carried out by NK. Sensor array was provided by DM, KB, and SS. NK wrote the first draft and BR revised it. The final draft submitted incorporated input from all authors and was approved by them.

The authors declare no competing financial interest.

\section{ACKNOWLEDGMENTS}

We would like to thank members of the Raman Lab for comments on previous versions of the manuscript. We would also like to thank Dr. Chunguang Jin for his help in designing and implementing our analyte delivery system. This work was funded by an Office of Naval Research grant (N00014-12-1-0089), NSF CAREER grant (1453022) and Children Discovery Institute's Interdisciplinary Research Initiative grants to B.R. 


\section{REFERENCES}

[1] M.L. Meade, C.R. Dillon, Signals and systems : models and behaviour, Signals and systems : models and behaviour, 2nd ed., Chapman \& Hall, London ; New York, 1991, pp. 73-5.

[2] M. Santiago, A. Pardo, F.A.M. Davide, C.D. Natale, A. D'Amico, A. Hierlemann, et al., Different strategies for the identification of gas sensing systems, Sensors and Actuators B: Chemical, 34(1996) 213-23.

[3] M. Holmberg, F.A.M. Davide, C. Di Natale, A. D'Amico, F. Winquist, I. Lundström, Drift counteraction in odour recognition applications: lifelong calibration method, Sensors and Actuators B: Chemical, 42(1997) 185-94.

[4] M. Holmberg, F. Winquist, I. Lundström, F. Davide, C. DiNatale, A. D'Amico, Drift counteraction for an electronic nose, Sensors and Actuators B: Chemical, 36(1996) 528-35.

[5] T.C. Pearce, Handbook of machine olfaction : electronic nose technology, Weinheim Germany: Wiley-VCH; 2003.

[6] D.C. Meier, B. Raman, S. Semancik, Detecting Chemical Hazards with TemperatureProgrammed Microsensors: Overcoming Complex Analytical Problems with Multidimensional Databases*, Annual Review of Analytical Chemistry, 2(2009) 463-84.

[7] B. Raman, J.L. Hertz, K.D. Benkstein, S. Semancik, Bioinspired Methodology for Artificial Olfaction, Analytical Chemistry, 8o(2008) 8364-71.

[8] R. Gutierrez-Osuna, Pattern analysis for machine olfaction: a review, Sensors Journal, IEEE, 2(2002) 189-202.

[9] J.W. Grate, Acoustic Wave Microsensor Arrays for Vapor Sensing, Chemical Reviews, $100(2000) 2627-48$.

[10] J. White, K. Truesdell, L.B. Williams, M.S. AtKisson, J.S. Kauer, Solid-State, DyeLabeled DNA Detects Volatile Compounds in the Vapor Phase, PLoS Biol, 6(2008) e9.

[11] D. Gopalakrishnan, W.R. Dichtel, Direct Detection of RDX Vapor Using a Conjugated Polymer Network, Journal of the American Chemical Society, 135(2013) 8357-62.

[12] B.R. Goldsmith, J.J. Mitala, J. Josue, A. Castro, M.B. Lerner, T.H. Bayburt, et al., Biomimetic Chemical Sensors Using Nanoelectronic Readout of Olfactory Receptor Proteins, ACS Nano, 5(2011) 5408-16.

[13] C.-J. Lu, J. Whiting, R.D. Sacks, E.T. Zellers, Portable Gas Chromatograph with Tunable Retention and Sensor Array Detection for Determination of Complex Vapor Mixtures, Analytical Chemistry, 75(2003) 1400-9.

[14] R. Banan Sadeghian, M. Saif Islam, Ultralow-voltage field-ionization discharge on whiskered silicon nanowires for gas-sensing applications, Nat Mater, 10(2011) 13540.

[15] G. Peng, U. Tisch, O. Adams, M. Hakim, N. Shehada, Y.Y. Broza, et al., Diagnosing lung cancer in exhaled breath using gold nanoparticles, Nat Nano, 4(2009) 669-73.

[16] P.T.T.B.C. Moseley, Solid-state gas sensors, Bristol; Philadelphia: A. Hilger; 1987.

[17] S. Semancik, R. Cavicchi, Kinetically Controlled Chemical Sensing Using Micromachined Structures, Accounts of Chemical Research, 31(1998) 279-87. 
[18] N.A. Rakow, K.S. Suslick, A colorimetric sensor array for odour visualization, Nature, 406(2000) 710-3.

[19] B.J. Doleman, N.S. Lewis, Comparison of odor detection thresholds and odor discriminablities of a conducting polymer composite electronic nose versus mammalian olfaction, Sensors and Actuators B: Chemical, 72(2001) 41-50.

[20] S.H. Lee, O.S. Kwon, H.S. Song, S.J. Park, J.H. Sung, J. Jang, et al., Mimicking the human smell sensing mechanism with an artificial nose platform, Biomaterials, 33(2012) 1722-9.

[21] C. Hagleitner, A. Hierlemann, D. Lange, A. Kummer, N. Kerness, O. Brand, et al., Smart single-chip gas sensor microsystem, Nature, 414(2001) 293-6.

[22] X. Jin, Y. Huang, A. Mason, X. Zeng, Multichannel Monolithic Quartz Crystal Microbalance Gas Sensor Array, Analytical Chemistry, 81(2008) 595-603.

[23] E. Martinelli, M. Santonico, G. Pennazza, R. Paolesse, A. D'Amico, C. Di Natale, Short time gas delivery pattern improves long-term sensor reproducibility, Sensors and Actuators B: Chemical, 156(2011) 753-9.

[24] M. Penza, M.A. Tagliente, L. Mirenghi, C. Gerardi, C. Martucci, G. Cassano, Tungsten trioxide $\left(\mathrm{WO}_{3}\right)$ sputtered thin films for a NOx gas sensor, Sensors and Actuators B: Chemical, 5o(1998) 9-18.

[25] B. Raman, D.C. Meier, J.K. Evju, S. Semancik, Designing and optimizing microsensor arrays for recognizing chemical hazards in complex environments, Sensors and Actuators B: Chemical, 137(2009) 617-29.

[26] B. Raman, R. Shenoy, D.C. Meier, K.D. Benkstein, C. Mungle, S. Semancik, Detecting and recognizing chemical targets in untrained backgrounds with temperature programmed sensors, Sensors Journal, IEEE, 12 (2012) 3238-47.

[27] Y. Hiranaka, T. Abe, H. Murata, Gas-dependent response in the temperature transient of $\mathrm{SnO}_{2}$ gas sensors, Sensors and Actuators B: Chemical, 9(1992) 177-82.

[28] X. Vilanova, E. Llobet, R. Alcubilla, J.E. Sueiras, X. Correig, Analysis of the conductance transient in thick-film tin oxide gas sensors, Sensors and Actuators B: Chemical, 31(1996) 175-80.

[29] E. Llobet, X. Vilanova, X. Correig, Novel technique to identify hazardous gases/vapors based on transient response measurements of tin oxide gas sensors conductance, Proc SPIE 2504, Environmental Monitoring and Hazardous Waste Site Remediation, Munich, Germany, 1995, pp. 559-66.

[30] A. Vergara, E. Llobet, J. Brezmes, P. Ivanov, C. Cané, I. Gràcia, et al., Quantitative gas mixture analysis using temperature-modulated micro-hotplate gas sensors: Selection and validation of the optimal modulating frequencies, Sensors and Actuators B: Chemical, 123(2007) 1002-16.

[31] C. Distante, M. Leo, P. Siciliano, K.C. Persaud, On the study of feature extraction methods for an electronic nose, Sensors and Actuators B: Chemical, 87(2002) 27488.

[32] S. Zhang, C. Xie, M. Hu, H. Li, Z. Bai, D. Zeng, An entire feature extraction method of metal oxide gas sensors, Sensors and Actuators B: Chemical, 132(2008) 81-9.

[33] E. Llobet, J. Brezmes, X. Vilanova, J.E. Sueiras, X. Correig, Qualitative and quantitative analysis of volatile organic compounds using transient and steady-state responses of 
a thick-film tin oxide gas sensor array, Sensors and Actuators B: Chemical, 41(1997) 13-21.

[34] G.J. Maclay, J.R. Stetter, S. Christesen, Use of time-dependent chemical sensor signals for selective identification, Sensors and Actuators, 20(1989) 277-85.

[35] G. Niebling, R. Müller, Non-linear signal evaluation with linear regression techniques for redundant signals, Sensors and Actuators B: Chemical, 25(1995) 805-7.

[36] A.K.M. Shafiqul Islam, Z. Ismail, M.N. Ahmad, B. Saad, A.R. Othman, A.Y.M. Shakaff, et al., Transient parameters of a coated quartz crystal microbalance sensor for the detection of volatile organic compounds (VOCs), Sensors and Actuators B: Chemical, 109(2005) 238-43.

[37] C. Martelli, J.R. Carlson, T. Emonet, Intensity Invariant Dynamics and Odor-Specific Latencies in Olfactory Receptor Neuron Response, The Journal of Neuroscience, 33 (2013) 6285-97.

[38] J.A. Riffell, E. Shlizerman, E. Sanders, L. Abrell, B. Medina, A.J. Hinterwirth, et al., Flower discrimination by pollinators in a dynamic chemical environment, Science, 344(2014) 1515-8.

[39] S.E. Stitzel, D.R. Stein, D.R. Walt, Enhancing Vapor Sensor Discrimination by Mimicking a Canine Nasal Cavity Flow Environment, Journal of the American Chemical Society, 125(2003) 3684-5.

[40] C.-Y. Su, C. Martelli, T. Emonet, J.R. Carlson, Temporal coding of odor mixtures in an olfactory receptor neuron, Proceedings of the National Academy of Sciences, 108(2011) 5075-80.

[41] N.J. Vickers, T.A. Christensen, T.C. Baker, J.G. Hildebrand, Odour-plume dynamics influence the brain's olfactory code, Nature, 410(2001) 466-70.

[42] D. Saha, K. Leong, C. Li, S. Peterson, G. Siegel, B. Raman, A spatiotemporal coding mechanism for background-invariant odor recognition, Nature Neuroscience, 16(2013) 1830-9.

[43] O. Mazor, G. Laurent, Transient Dynamics versus Fixed Points in Odor Representations by Locust Antennal Lobe Projection Neurons, Neuron, 48(2005) 661-73.

[44] R.F. Galán, S. Sachse, C.G. Galizia, A.V.M. Herz, Odor-Driven Attractor Dynamics in the Antennal Lobe Allow for Simple and Rapid Olfactory Pattern Classification, Neural Computation, 16(2004) 999-1012.

[45] T. Artursson, T. Eklöv, I. Lundström, P. Mårtensson, M. Sjöström, M. Holmberg, Drift correction for gas sensors using multivariate methods, Journal of Chemometrics, 14(2000) 711-23.

[46] A. Hierlemann, R. Gutierrez-Osuna, Higher-Order Chemical Sensing, Chemical Reviews, 108(2008) 563-613.

[47] A. Vergara, S. Vembu, T. Ayhan, M.A. Ryan, M.L. Homer, R. Huerta, Chemical gas sensor drift compensation using classifier ensembles, Sensors and Actuators B: Chemical, 166-167(2012) 320-9.

[48] M. Zuppa, C. Distante, P. Siciliano, K.C. Persaud, Drift counteraction with multiple self-organising maps for an electronic nose, Sensors and Actuators B: Chemical, 98(2004) 305-17. 
[49] D. Saha, C. Li, S. Peterson, W. Padovano, N. Katta, B. Raman, Behavioural correlates of combinatorial versus temporal features of odour codes, Nat Commun, 6(2015).

[5o] J. Murlis, J.S. Elkinton, R.T. Cardé, Odor Plumes and How Insects Use Them, Annual Review of Entomology, 37(1992) 505-32.

[51] K. Benkstein, C. Martinez, G. Li, D. Meier, C. Montgomery, S. Semancik, Integration of nanostructured materials with MEMS microhotplate platforms to enhance chemical sensor performance, J Nanopart Res, 8(2006) 809-22.

[52] C.J. Martinez, B. Hockey, C.B. Montgomery, S. Semancik, Porous Tin Oxide Nanostructured Microspheres for Sensor Applications, Langmuir, 21(2005) 7937-44.

[53] S. Semancik, R.E. Cavicchi, M.C. Wheeler, J.E. Tiffany, G.E. Poirier, R.M. Walton, et al., Microhotplate platforms for chemical sensor research, Sensors and Actuators B: Chemical, 77(2001) 579-91.

[54] A.G. Shirke, R.E. Cavicchi, S. Semancik, R.H. Jackson, B.G. Frederick, M. Clayton Wheeler, Femtomolar isothermal desorption using microhotplate sensors, Journal of Vacuum Science \& Technology A, 25(2007) 514-26.

[55] R.E. Cavicchi, S. Semancik, F. DiMeo, Jr., C.J. Taylor, Featured Article: Use of Microhotplates in the Controlled Growth and Characterization of Metal Oxides for Chemical Sensing, Journal of Electroceramics, 9(2002) 155-64.

[56] P.H. Rogers, S. Semancik, Development of optimization procedures for applicationspecific chemical sensing, Sensors and Actuators B: Chemical, 163(2012) 8-19.

[57] Z. Boger, D.C. Meier, R.E. Cavicchi, S. Semancik, Rapid Identification of Chemical Warfare Agents by Artificial Neural Network Pruning of Temperature-Programmed Microsensor Databases, Sensor Letters, 1(2003) 86-92.

[58] W.M. Sears, K. Colbow, F. Consadori, Algorithms to improve the selectivity of thermally-cycled tin oxide gas sensors, Sensors and Actuators, 19(1989) 333-49.

[59] J. Li, Y. Lu, Q. Ye, M. Cinke, J. Han, M. Meyyappan, Carbon Nanotube Sensors for Gas and Organic Vapor Detection, Nano Letters, 3(2003) 929-33.

[6o] L. Ljung, System Identification-Theory for the User, 2nd editionPTR Prentice Hall, Upper Saddle River, NJ, (1999).

[61] G.T. Buračas, G.M. Boynton, Efficient Design of Event-Related fMRI Experiments Using M-Sequences, NeuroImage, 16(2002) 801-13.

[62] A. Vergara, E. Llobet, J. Brezmes, X. Vilanova, P. Ivanov, I. Gracia, et al., Optimized temperature modulation of micro-hotplate gas sensors through pseudorandom binary sequences, Sensors Journal, IEEE, 5(2005) 1369-78.

[63] A. Ziyatdinov, J. Fonollosa, L. Fernández, A. Gutierrez-Gálvez, S. Marco, A. Perera, Bioinspired Early Detection through Gas Flow Modulation in Chemo-Sensory Systems, Sensors and Actuators B: Chemical, 206(2015) 538-47.

[64] H. Suzuki, Antennal movements induced by odour and central projection of the antennal neurones in the honey-bee, Journal of Insect Physiology, 21(1975) 831-47.

[65] D.G. Laing, Identification of single dissimilar odors is achieved by humans with a single sniff, Physiology \& Behavior, 37(1986) 163-70.

[66] M.D. Woodka, B.S. Brunschwig, N.S. Lewis, Use of Spatiotemporal Response Information from Sorption-Based Sensor Arrays to Identify and Quantify the Composition of Analyte Mixtures, Langmuir, 23(2007) 13232-41. 
[67] A. Vergara, K.D. Benkstein, C.B. Montgomery, S. Semancik, Demonstration of Fast and Accurate Discrimination and Quantification of Chemically Similar Species Utilizing a Single Cross-Selective Chemiresistor, Analytical Chemistry, 86(2014) 6753-7.

[68] R.E. Cavicchi, J.S. Suehle, K.G. Kreider, M. Gaitan, P. Chaparala, Fast temperature programmed sensing for micro-hotplate gas sensors, Electron Device Letters, IEEE, 16(1995) 286-8.

[69] R.E. Cavicchi, J.S. Suehle, K.G. Kreider, M. Gaitan, P. Chaparala, Optimized temperature-pulse sequences for the enhancement of chemically specific response patterns from micro-hotplate gas sensors, Sensors and Actuators B: Chemical, 33(1996) 142-6.

[7o] R. Gosangi, R. Gutierrez-Osuna, Active Temperature Programming for Metal-Oxide Chemoresistors, Sensors Journal, IEEE, 10(2010) 1075-82.

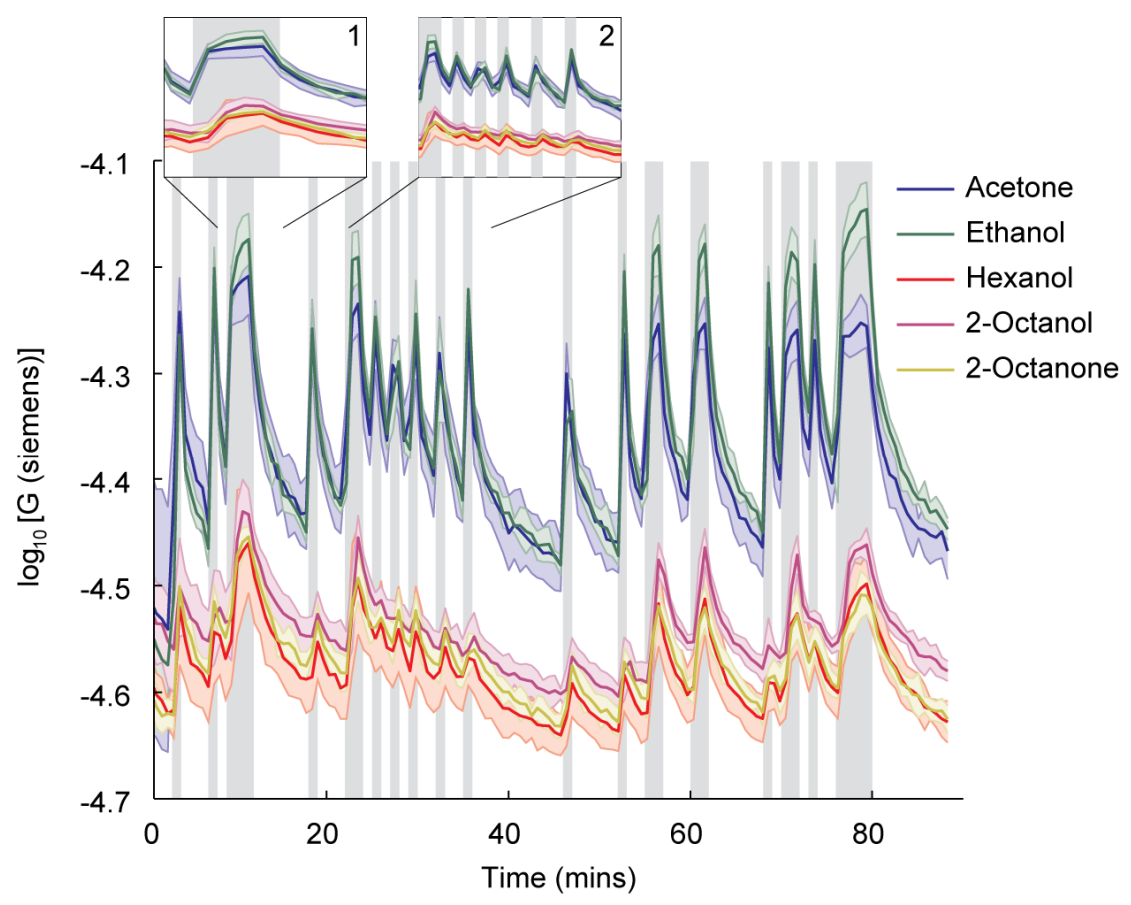

Figure 1. Responses of a metal-oxide sensor $\left(\mathrm{SnO}_{2}\right)$ to five different analytes presented in pulsatile fashion. Each trace represents the mean sensor response to an analyte with the sensor operating at $435{ }^{\circ} \mathrm{C}$. Color bands represent standard deviations. Gray bars indicate periods when the sensor was exposed to the analyte. First inset reveals response onset differences during a prolonged single pulse. Second inset reveals diminishing of responses when probed with short, back-to-back stimulus pulses. 
A)
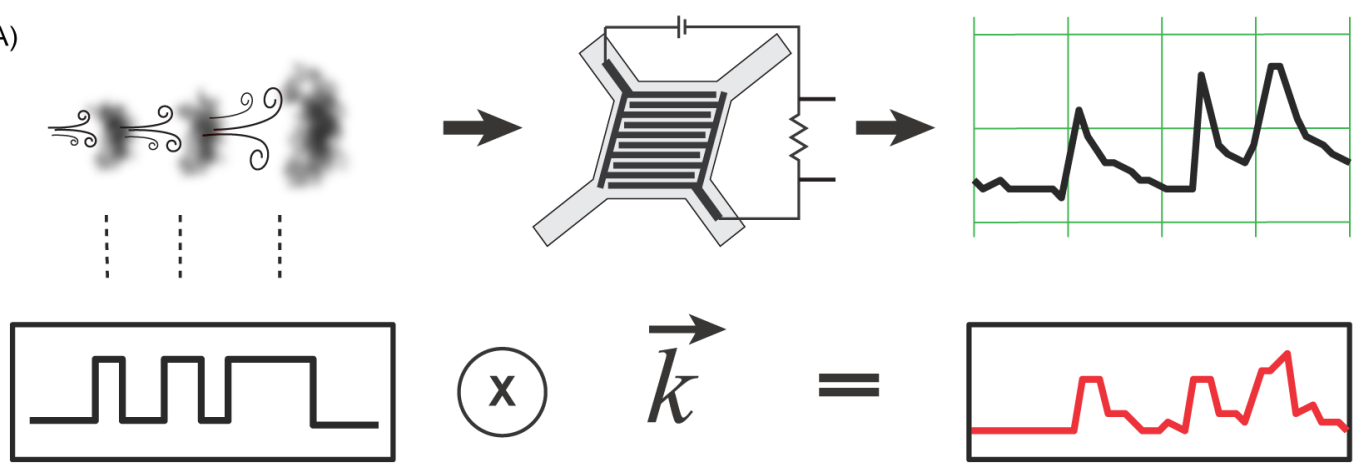

B)
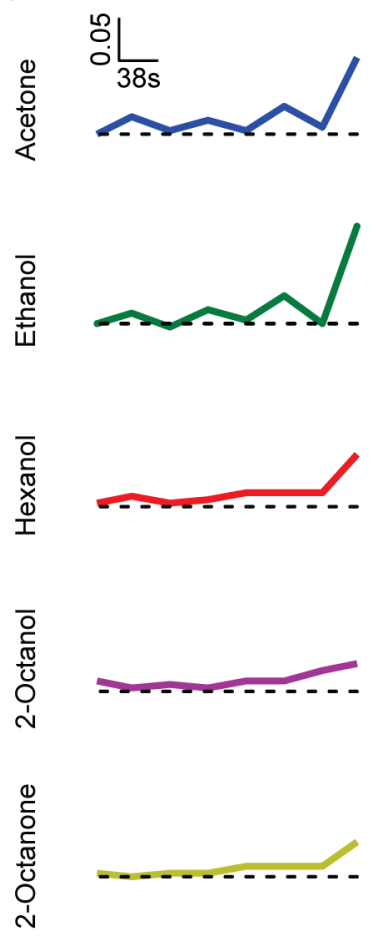

D)

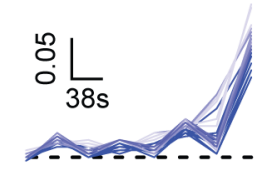

Acetone
C)
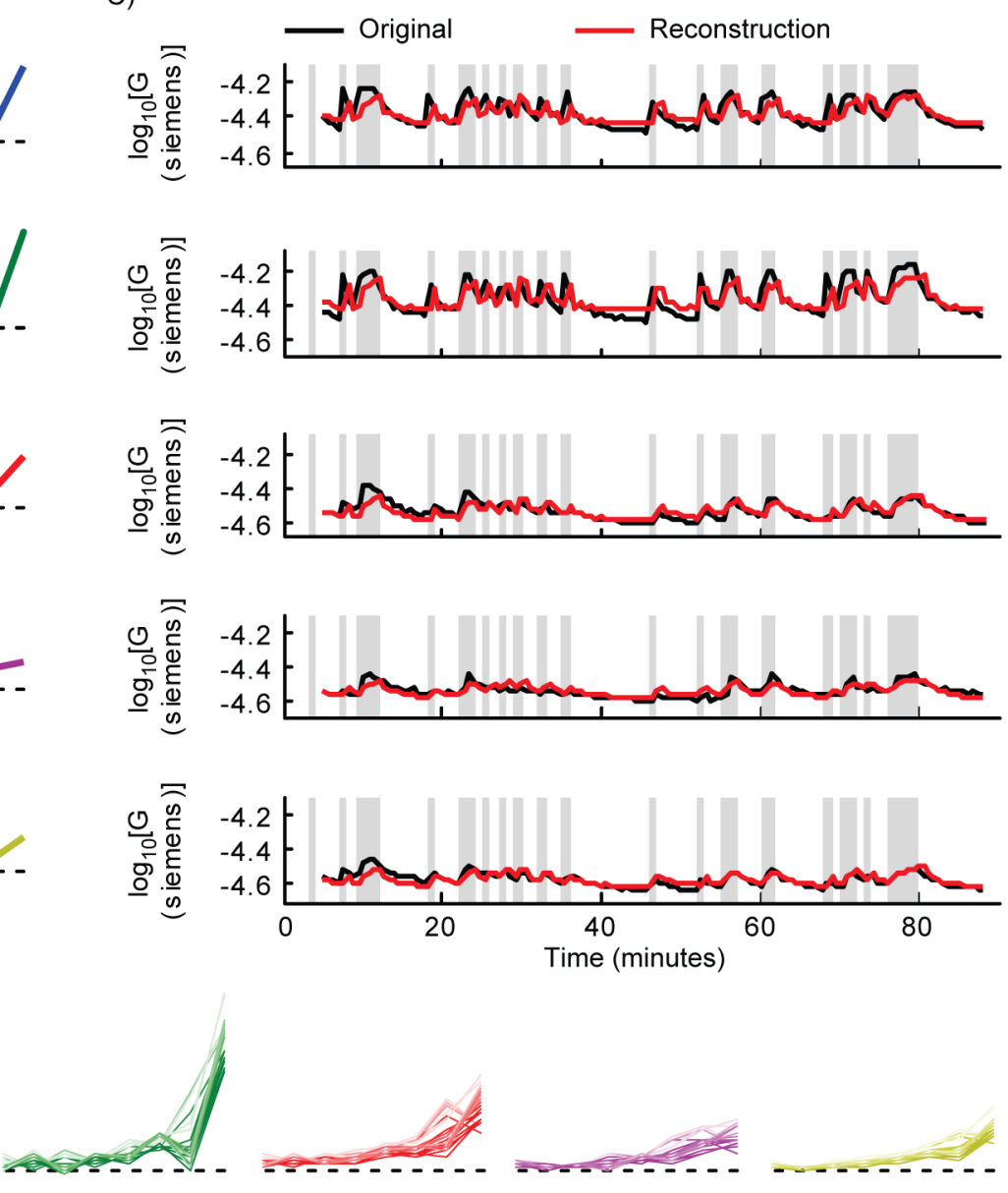

Ethanol

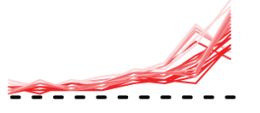

Hexanol

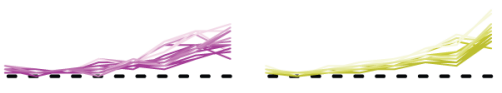

2-Octanol

2-Octanone

Figure 2. I/O transforms of a metal-oxide chemiresistor. A) Top row) Schematic illustration of the proposed approach. An analyte is pulsed over the sensor and causes changes in resistance across a metal oxide sensing film. Bottom row) A schematic of a sensor response reconstruction is shown. Given a specific stimulus sequence, the sensor's operation is akin to a mathematical transformation that is specific for a given analyte. Convolving the analyte-specific filter with the most recent stimulus history will generate a prediction for the sensor's response that can be expected at the end of that period. B) I/O transforms $(\vec{k})$ generated using sensor's response at $435{ }^{\circ} \mathrm{C}$ for each of the five analytes examined in the study. C) Comparison of the sensor's actual response at $435{ }^{\circ} \mathrm{C}$ (black) and reconstructions (red) obtained for all five analytes. D) I/O transformations for each analyte at all twenty-eight temperatures used (see Fig. S-1). Higher intensity of color indicates higher temperature. 
A)
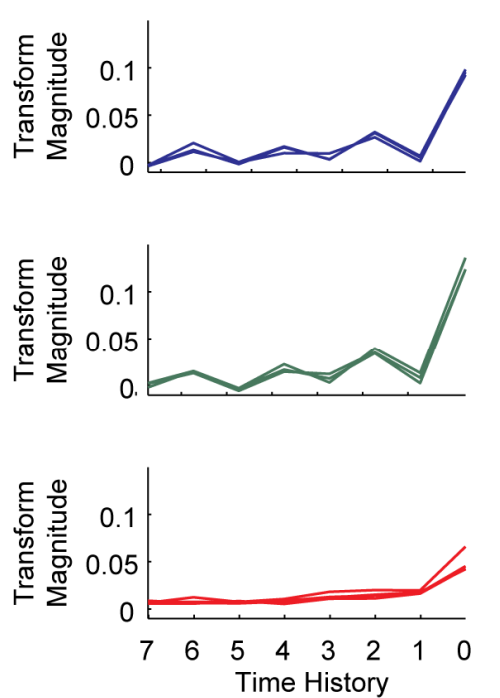
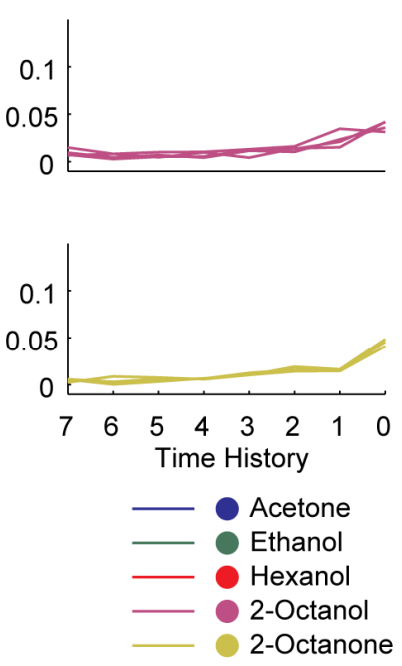

B)

Figure 3. I/O transforms for different analytes are consistent across different training trials. A) I/O transforms obtained using sensor response at $435^{\circ} \mathrm{C}$ are shown for each analyte and for each training run. B) Visualization of the I/O transforms $(\vec{k})$ of different analytes and for different runs using principle component analysis is shown.

A)
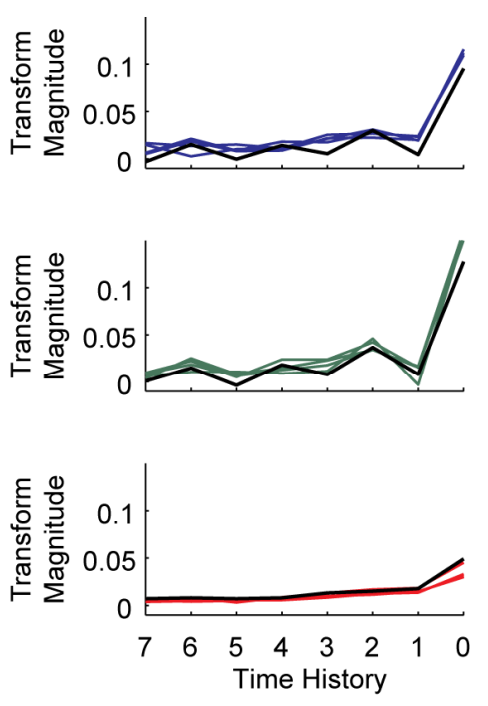

B)
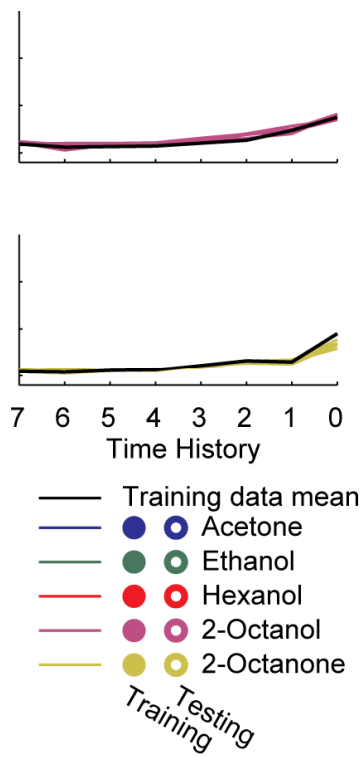

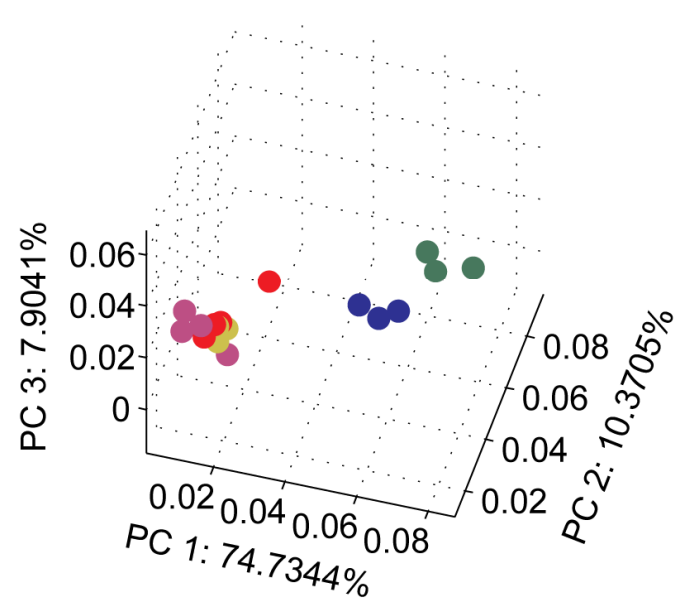

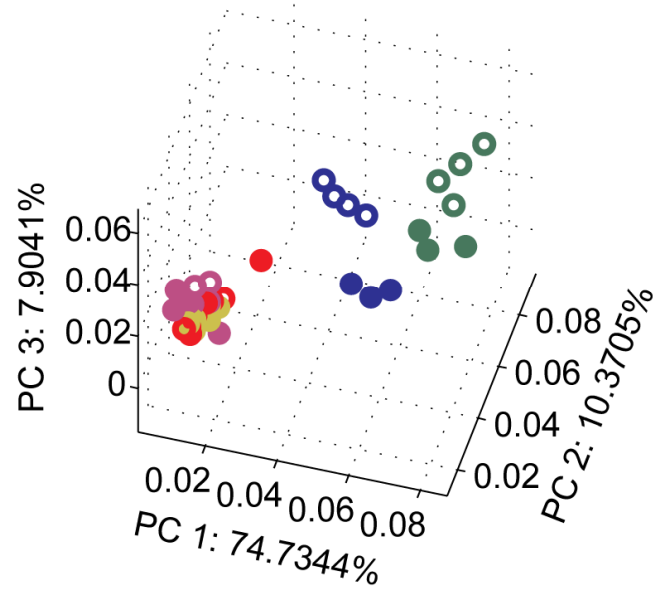

Figure 4. I/O transforms are robust with respect to sensor aging. A) All I/O transforms of sensor responses at 435 ${ }^{\circ} \mathrm{C}$ after aging are shown. Black line represents mean transforms obtained from pre-aged sensor (i.e. training data). Each colored line represents I/O transform obtained for an individual test phase trial. B) Principle component analysis of I/O transforms before (filled symbols) and after (open symbols) sensor aging. Only training data was used to calculate the principal component axes. 
A)

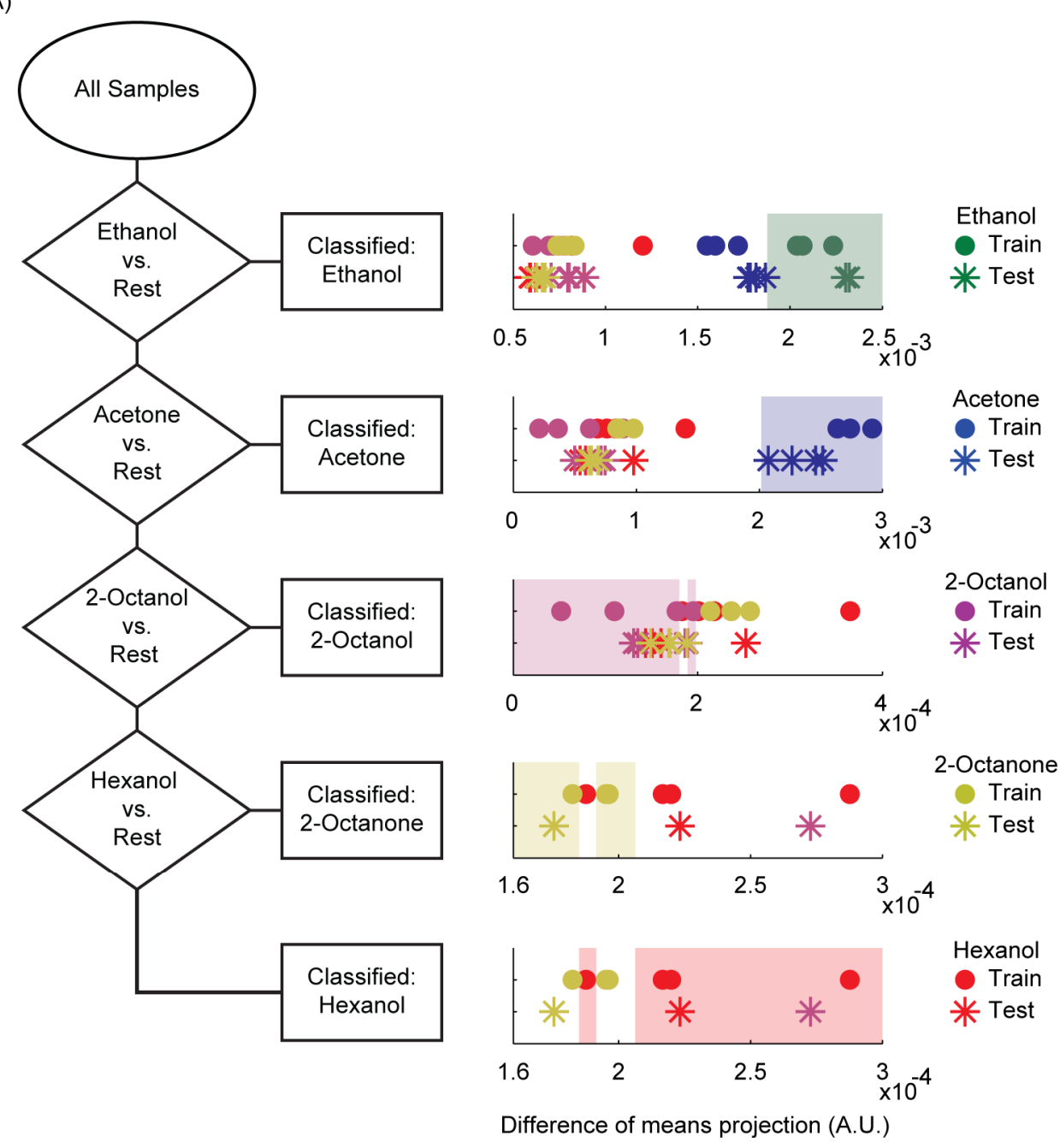

B)

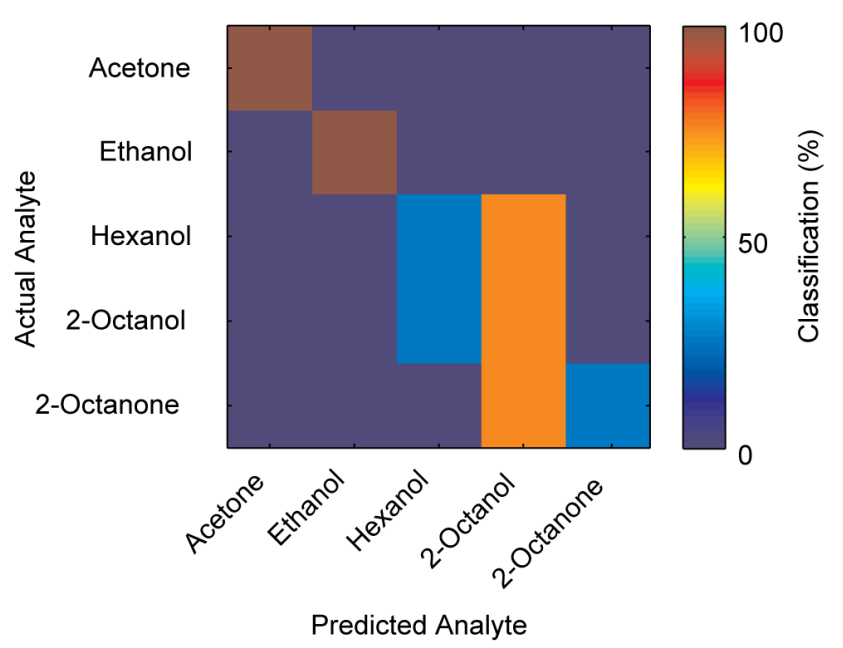

Figure 5. A hierarchical classification algorithm for multi-analyte recognition. A) Hierarchical classification algorithm used to identify analytes is schematically shown. At each level, both training and testing data were projected onto the differences of means plane between the most distinct/farthest class and its nearest neighboring class. The data after this projection is shown in the panels on the right. The regions where the class assignment favored the distant class are identified in each subplot displaying projected data. When testing data projects onto uncolored regions, those points will move down a level in order to be precisely classified. B) Confusion matrix quantitatively summarizing the performance of the hierarchical classification approach is shown. 
A)
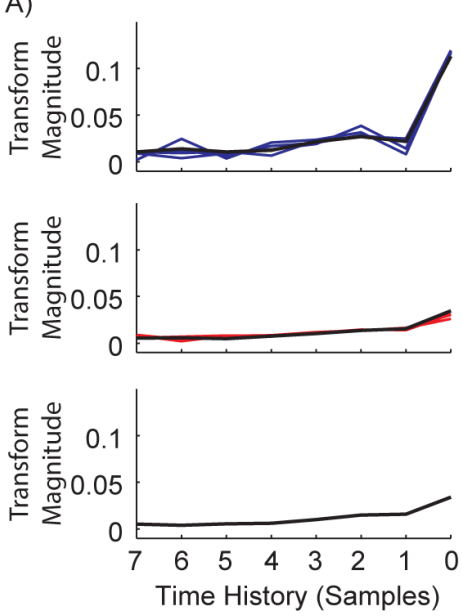

C)

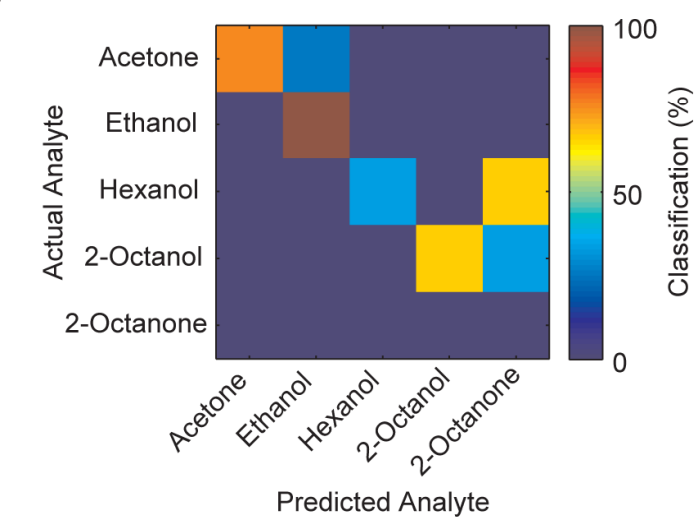

B)
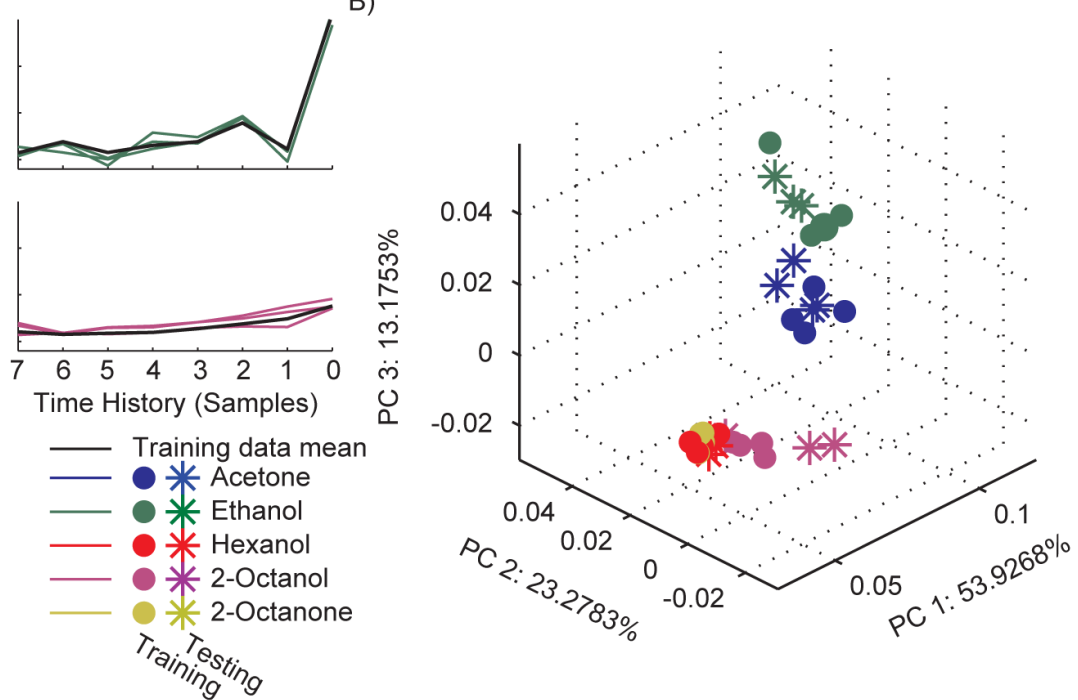

Figure 6. I/O transforms are robust with respect to analyte concentration. A) Each panel reveals I/O transforms estimated from a sensor's responses at $435{ }^{\circ} \mathrm{C}$ to particular analyte presented at varying concentrations. Black line represents mean transforms obtained from sensor response to a fixed concentration (i.e. training data). Each colored line represents an I/O transform obtained for an individual test phase trial. B) Visualization of I/O transforms obtained at varying concentrations of analytes are shown (filled symbols - training datapoints; asterisks - test datapoints). Only training data were used to calculate the principle component axes. C) Confusion matrix quantifying classification performance. 

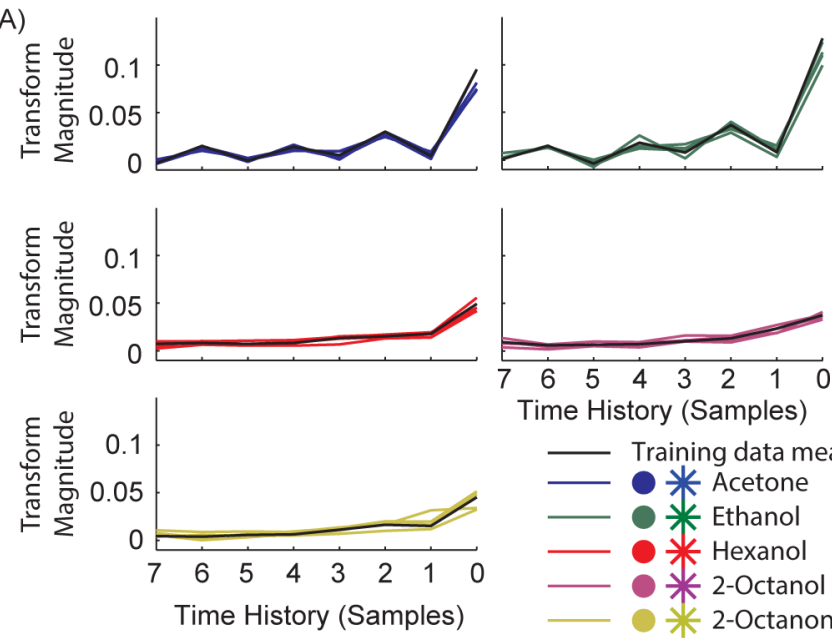

Time History (Samples)

_ Training data mean * Acetone

- Ethanol

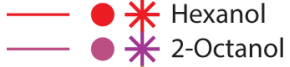

* 2-Octanone Training $_{\text {Testing }}$

C)

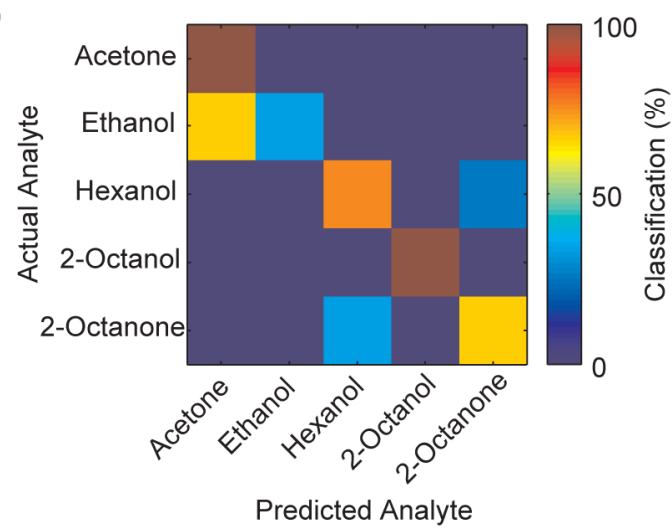

B)

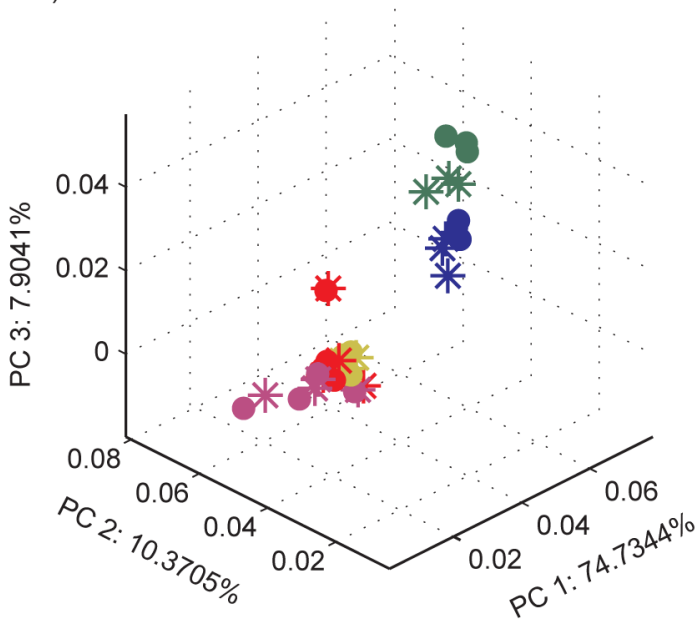

Figure 7. I/O transforms are robust across sensors of similar manufacture. A) All I/O transforms of sensor 2 response at $435{ }^{\circ} \mathrm{C}$ for are shown. Black line represents mean transforms obtained from sensor 1 response (i.e. training data). Each colored line represents an I/O transform obtained for an individual test phase trial. B) Principle component analysis of I/O transforms of sensor 1 (filled symbol) and sensor 2(asterisks). Only training data was used to calculate the principle component axes. C) Confusion matrix quantifying classification performance. 

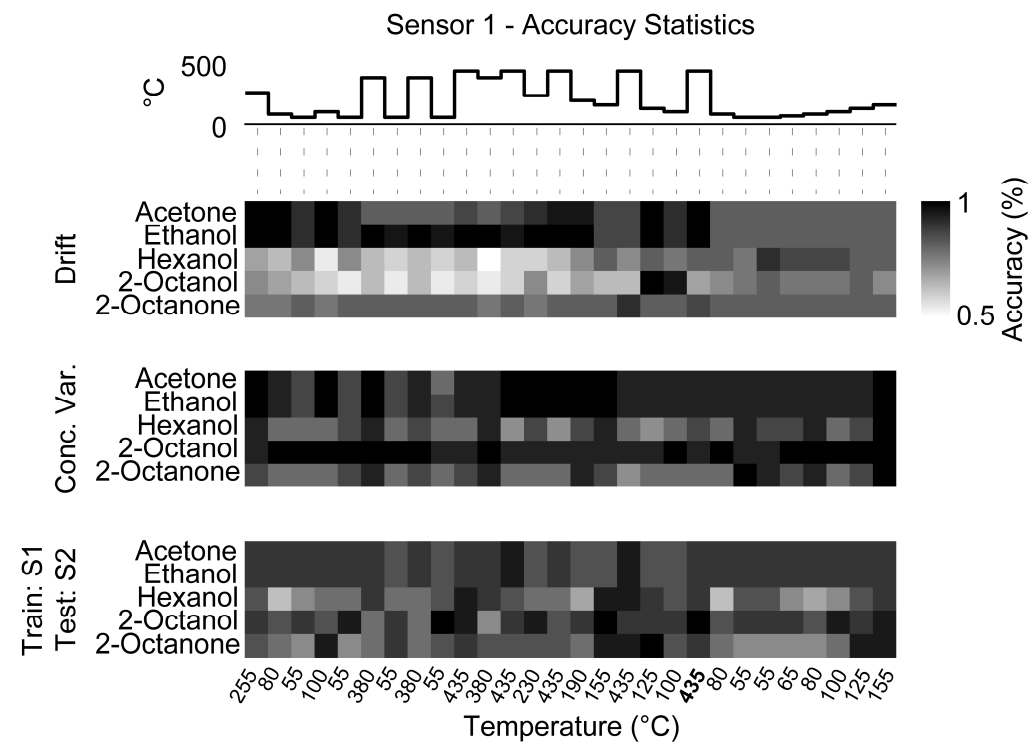

Figure 8. Classification performance is temperature and hysteresis dependent. Classification accuracy for sensor 1 is shown for each temperature in the cycle for the three conditions examined (from top to bottom): (2 to 3) months drift, for varying concentrations, and across different sensors. The temperature cycle used in the study is shown at the top of the plot. In general, for all three cases, the classification performance is better in the second half of the temperature cycle. 
A)
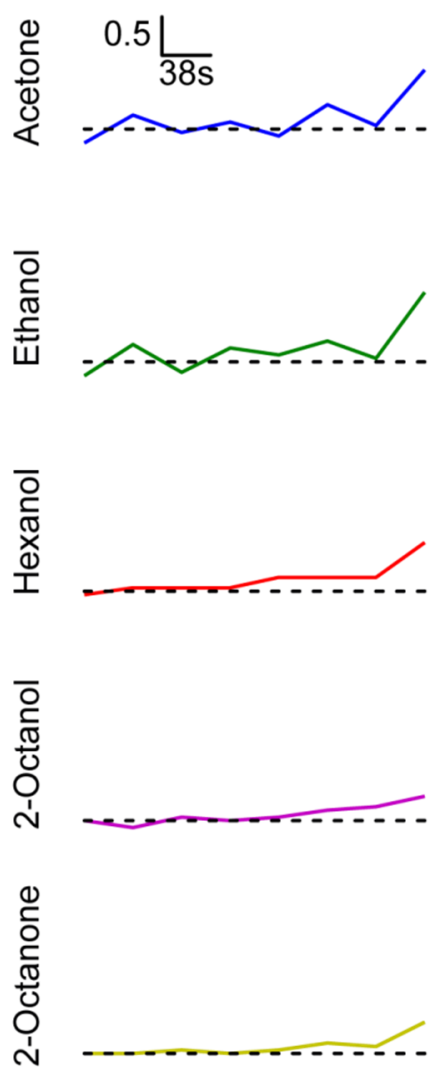

C)

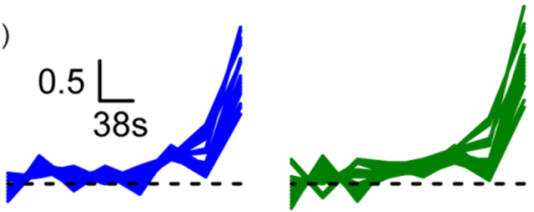

B)
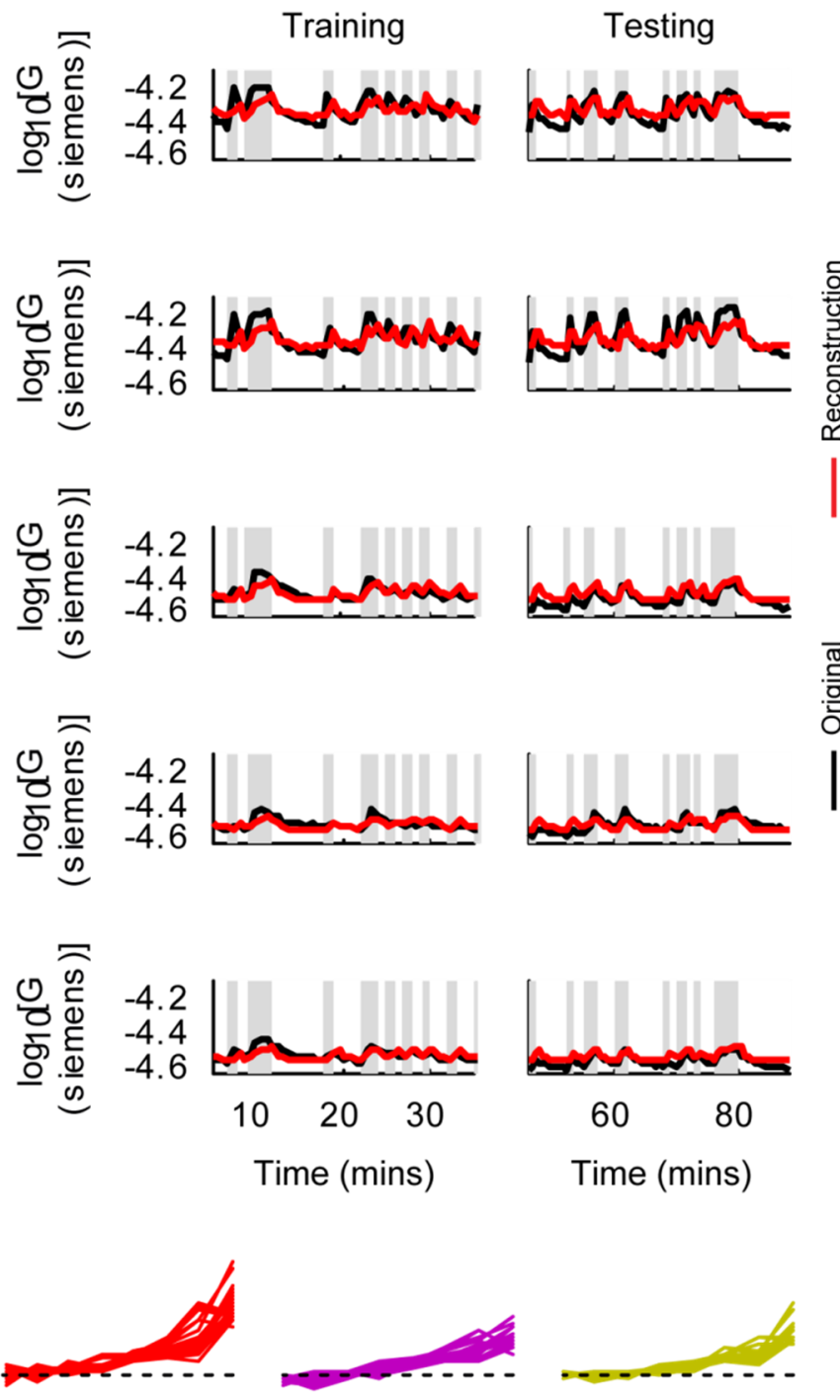

Figure 9. I/O transforms of a metal-oxide chemiresistor. A) I/O transforms $(\vec{k})$ generated using sensor's response at $435{ }^{\circ} \mathrm{C}$ for each of the five analytes are shown. Only half the entire stimulus sequence used in Fig. 2 used to generate these transforms. B) Comparison between the sensor's actual response at $435{ }^{\circ} \mathrm{C}$ (black) and reconstructions (red) obtained for all five analytes for the first half (training) and second half (testing) of the stimulus sequence are provided. Note that the stimulus sequences during training and testing portions are different. C) I/O transformations for each analyte at all twenty-eight temperatures used are shown. 


\section{VITAE}

Nalin Katta received his B.S. degree in biomedical engineering from the Washington University in St. Louis, in 2010. He is a Ph.D. candidate in the Department of Biomedical Engineering at Washington University, St. Louis, MO, under the guidance of Dr. Baranidharan Raman. His current research interests include sensory and systems neuroscience, bio-mimetic sensors, and device design.

Douglas C. Meier received the B.A. degree in chemistry from Northwestern University, Evanston, IL, and the Ph.D. degree in chemistry from Texas A\&M University, College Station, where he studied the chemical physics of model catalyst systems under the guidance of Prof. D. W. Goodman. He is currently a National Institute of Standards and Technology (NIST) Research Chemist with the Process Sensing Group, NIST, Gaithersburg, MD, applying surface chemistry and thin-film science in the development of advanced chemical microsensor arrays. Dr. Meier was awarded a National Research Council Post-Doctoral Research Associateship from the Process Sensing Group, NIST.

Kurt D. Benkstein received his B.S. degree in Chemistry in 1995 from Iowa State University and his M.S. and Ph.D. degrees in Chemistry from Northwestern University in 1996 and 2000, respectively. He went to the National Renewable Energy Laboratory in 2000 as a postdoctoral researcher to study the relation between film morphology and electron transport in dye-sensitized nanoparticle solar cells. In 2003, Dr. Benkstein joined the National Institute of Standards and Technology as a Research Chemist to study nanostructured materials for chemical sensors.

Steve Semancik is the Project Leader of the Chemical and Bioanalytical Microsensor Program at the National Institute of Standards and Technology (NIST) in Gaithersburg, Maryland. He received his B.S. degree in physics from Rensselaer Polytechnic Institute and his Sc.M. and Ph.D. degrees, also in physics, from Brown University. Dr. Semancik's professional research career began as a National Research Council Postdoctoral Fellow, and has been centered in the fields of surface science and sensor science. His recent work has focused on developing improved nanomaterials for chemical and biochemical sensing, and combining such high performance materials with micromachined platforms to realize advanced microsensor devices and operating modes. He has authored or coauthored more than 150 papers, including multiple reviews, several book chapters, and six patents. Dr. Semancik is an elected Fellow of both the American Physical Society and the American Vacuum Society, has served as a Member of the Editorial Board of two sensor journals, and is a Member of the Steering Committee of the International Meeting on Chemical Sensors.

Baranidharan Raman received the B.Sc. Eng. degree (with distinction) in computer science from the University of Madras, Chennai, India, in 2000, and the M.S. and Ph.D. degrees in computer science from Texas A\&M University, College Station, TX, in 2003 and 2005, respectively. He is an Assistant Professor with the Department of Biomedical Engineering, Washington University, St. Louis, MO. From 2006 to 2010, he was a joint Post-Doctoral Fellow with the National Institutes of Health and the National Institute of Standards and Technology, Gaithersburg, MD. His current research interests include sensory and systems neuroscience, sensor-based machine olfaction, machine learning, biomedical intelligent systems, and dynamical systems. Dr. Raman is a recipient of the Wolfgang Gopel Award in 2011 from the International Society for Olfaction and Chemical Sensing and a NSF CAREER awardee. 